not due to nutritional, cardiac, or renal disease. It is neither inflammatory nor necessarily associated with inflammation of the joints of the feet and ankles. It is not a lymphoedema. It is not painless, but is uncomfortable, tender, or painful. It is not always pitting, becoming solid in the later stages. There is considerable evidence to suggest that this is a venous oedema.

\section{Summary}

Forty patients with rheumatoid arthritis and oedema have been investigated to find the cause of the oedema. Specimens of oedema fluid from 18 of these have been analysed. The oedema is a low-protein oedema, and the protein level bears no relation to duration and no relation to the protein level of serum. There is no relation to inflammation of the joints of the feet and ankles, and no relation to nutritional, cardiac, or renal disease. Varicose veins and thrombophlebitis are not the cause of the oedema. The natural history of this phenomenon suggests that it is a venous oedema, and this is supported by the appearance of the skin of the feet and ankles, and by the oedema-fluidprotein levels.
The explanation given by Hollander is incorrect. Oedema of the feet and ankles should be regarded as a clinical feature of rheumatoid arthritis due to altered venous function.

I should like to thank Professors S. J. Hartfall, A. Hemingway, G. H. Lathe, and D. R. Wood for their advice, interest, and support.

\section{REFERENCES}

Bennhold, H. (1957). Uber Zwei Fälle von Analbuminaemiae. Protides of the Biological Fluids, edited by H. Peeters, p. 223. Elsevier, London.

Burn, J. H. (1962). Drugs, Medicine, and Man, p. 47. Allen and Unwin, ondon.

Crockett, D. J. (1956). Lancet, 2, 1179

Drinker, C. K., Field, M. E., Heim, J. W., and Leigh, O. C., jun. (1934). Amer. F. Physiol., 109, 572 .

Exton-Smith, A. N., and Crockett, D. J. (1957). Brit. med. F., 2, 1280.

Hendry, E. B. (1962). Clin. Chem., 8, 246.

Hollander, J. L. (1960). Arthritis and Allied Conditions, 6th ed., p. 40. Kimpton, London.

Lancet, 1960, 2, 1179.

Park, D. C., and Swinburne, K. (1964). Brit. med. F., 1, 86.

Samuels, S. S. (1956). Diagnosis and Treatment of Vascular Disorders, Chap. 22, p. 556. Williams and Wilkins, Baltimore.

Stead, E. A., jun., and Warren, J. V. (1944). f. clin. Invest., 23, 283.

Taylor, G. W., Kinmonth, J. B., and Dangerfield, W. G. (1958). Brit. med. F., 1, 1159 .

\title{
Smallpox Vaccination in Pregnancy: A Prospective Study
}

\author{
GEOFFREY J. BOURKE,* M.A., M.B., D.P.H., D.C.H. ; RICHARD J. WHITTY,* L.R.C.P.I., D.C.H.
}

Ever since the observation by Gregg (1941) of a high incidence of congenital defects of the eye in children born to women who had had rubella in pregnancy, interest has been stimulated in infections as probable aetiological factors in congenital abnormality or pregnancy wastage. It follows that a similar interest should be taken in the artificial introduction into the body of virus or bacterial products during immunization procedures in pregnancy.

A smallpox outbreak occurred in England and Wales between December 1961 and April 1962. As a consequence of press, radio, and television publicity, the staffs of public health clinics and other doctors in Dublin City received many requests for vaccination against smallpox, and large numbers of people were vaccinated as a result. Despite notices displayed at public health clinics stating that routine smallpox vaccination was contraindicated in pregnancy, it was inevitable that some pregnant women would be vaccinated, and this paper reports a study of the effects on the foetus of smallpox vaccination.

\section{Method}

The population of Dublin City is served by four maternity hospitals, which together account for over $80 \%$ of the births of the city. During the period 2 April to 3 September 1962 of the city. During the peran attending the antenatal clinics of these four hospitals was asked whether or not she had been vaccinated against smallpox during her current pregnancy. If the answer was in the affirmative she was asked for the date and place of vaccination, and was interviewed to determine whether vaccination had been successful or not. Because it was necessary to rely on the patient's statement to assist in interpretation of

- From the Department of Social Medicine, Trinity College, Dublin. successful reactions it was decided to use a history of vesicle ("water blister") which later formed a pustule (" pus blister") and the presence of a recent scar as the criteria of successful vaccination. If the woman had not been vaccinated she was advised against the procedure. The appropriate facts were recorded on each chart.

It should be noted that the woman was not asked direct questions about the reaction but was invited to describe the lesion which appeared on the arm after vaccination. It is realized that omission of the immediate-type reactions will leave in the study only those with the more severe types of vaccination reaction. Consequently the cases we are dealing with are those in which the insult, if any, to the foetus following smallpox vaccination is greatest.

Twelve months later the chart of each vaccinated subject was obtained; the four adjacent charts, two on each side of the index chart, of women who were recorded as being unvaccinated during their pregnancy, and whose first visit to the antenatal clinic was on the same day as the vaccinated subject, were taken as controls. There were, then, four unvaccinated controls for each vaccinated case. It must be mentioned, of course, that, though the unvaccinated had been advised against vaccination, it is not definitely known whether or not they remained unvaccinated throughout the pregnancy. However, where a congenital abnormality, stillbirth, neonatal death, or abortion was recorded among the controls, a home visit was made to inquire whether smallpox vaccination had in fact been done, but none of these controls was found to have been vaccinated during the remainder of the pregnancy. The following facts were recorded from each chart: age, parity, blood group, duration of pregnancy in weeks, the outcome of the pregnancy (whether abortion, stillbirth, neonatal death, congenital abnormality, or normal live birth), the weight and sex of the 
infant, and, for each vaccinated woman, the stage of the pregnancy in weeks when vaccination was performed.

\section{Results}

During the 22 weeks when patients were asked whether or not they had been vaccinated on the current pregnancy, 117 reported that vaccination had been done. Five women who did not have successful vaccination according to the standards adopted were excluded from the study, leaving for observation 112 mothers successfully vaccinated during pregnancy. The five mothers omitted from the study all produced healthy living children at term; the remaining 112 women gave birth to 113 infants (two pairs of twins) and one abortion. There were 448 control mothers, who gave birth to 449 infants (five pairs of twins) and four abortions. Analysis shows that the vaccinated and control groups are very similar with regard to age, parity, and blood-group distribution. The average age of the vaccinated women was 28.11 years and of the controls 28.23 years ; the average parity of the vaccinated was 2.68 and of the controls 2.66. No significant difference was observed in the distribution of the $\mathrm{ABO}$ and rhesus blood groups among the vaccinated and the controls $\left(\chi^{2}=3.692 ; n=7 ; 0.90>P>0.80\right)$. Further, when the stage of pregnancy at the first visit is analysed by trimester no significant difference is found between the vaccinated and the controls $\left(\chi^{2}=0.478 ; \mathrm{n}=2 ; 0.80>\mathrm{P}>0.70\right)$. For the purpose of this study all estimations of the length of pregnancy are calculated in weeks and date from the first day of the last menstrual period.

Among the women vaccinated during pregnancy there were three stillbirths with no apparent abnormality, one abortion at 26 weeks, three congenital defects (one of these was stillborn), and 107 normal live births. The abnormalities were hydrocephalus with spina bifida, spina bifida alone, and Down's syndrome; the mothers were vaccinated when 23,4 , and 27 weeks pregnant respectively. The mothers of the normal stillbirths were all vaccinated within the second trimester, when 15, 17, and 21 weeks pregnant. The mother who aborted at 26 weeks was vaccinated when 10 weeks pregnant. One neonatal death occurred in a twin, premature by weight, whose mother was vaccinated when 11 weeks pregnant; the other twin was alive and healthy. Fifty-four women were vaccinated when in the first trimester of pregnancy (11 under 4 weeks and 43 in pregnancies of 4-12 weeks' duration), 40 mothers were vaccinated at 12-24 weeks' pregnancy, and 18 at over 24 weeks.

Among the controls there were six stillbirths with no apparent abnormality, four abortions, three live births with congenital defects (spina bifida with hydrocephalus, cleft palate, and spina bifida alone), and 440 normal live births. The four abortions occurred at $6,7,12$, and 16 weeks of pregnancy. In addition, there were four neonatal deaths (two infants premature by weight and one post-mature infant ; the fourth neonatal death was that of the baby with spina bifida and hydrocephalus already referred to).

It will be seen from the Table that the abortions, congenital deformities, and normal stillbirths among the mothers vac-

\begin{tabular}{|c|c|c|c|c|c|c|}
\hline $\begin{array}{l}\text { Vaccinal State } \\
\text { of Women }\end{array}$ & & Stillbirths & Abortions & $\begin{array}{c}\text { Congenital } \\
\text { Defects }\end{array}$ & $\begin{array}{l}\text { Normal } \\
\text { Births }\end{array}$ & Total \\
\hline $\begin{array}{l}\text { Vaccinated } \\
\text { Unvaccinated }\end{array}$ & 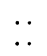 & $\begin{array}{l}3 \\
6\end{array}$ & $\begin{array}{l}1 \\
4\end{array}$ & $\begin{array}{l}3^{*} \\
3^{*}\end{array}$ & $\begin{array}{l}107 \\
440\end{array}$ & $\begin{array}{l}114 \\
453\end{array}$ \\
\hline Total & $\ldots$ & 9 & 5 & 6 & 547 & 567 \\
\hline
\end{tabular}

- One congenital defect also stillbirth, but recorded here as congenital defect only

cinated and those unvaccinated in the course of their pregnancies do not differ significantly in their distribution.
Duration of Pregnancy.-This did not differ significantly among the vaccinated women (39.99 weeks) and the controls (39.88 weeks). Further, when the vaccinated women are divided into trimesters according to the stage of pregnancy when vaccination took place, no significant differences are demonstrable between the length of pregnancy of those vaccinated within the first 12 weeks (39.88 weeks), 12-24 weeks (40.18 weeks), or over 24 weeks (39.94 weeks) and the controls (39.88 weeks).

Weight of Infant at Birth.-The weights of infants at birth did not differ significantly among the vaccinated women (7.65 lb. ; 3,470 g.) and controls (7.57 lb. ; 3,435 g.). Again, when the vaccinated women are divided into trimesters according to the stage of pregnancy when vaccination was performed, no significant differences are apparent between the weights of infants at birth when the mother was vaccinated within the first 12 weeks (7.51 lb. ; 3,405 g.), 12-24 weeks (7.90 lb.; 3,585 g.), or over 24 weeks (7.28 lb. ; 3,300 g.) or controls $(7.57 \mathrm{lb} . ; 3,435 \mathrm{~g}$.$) . The incidence of prematurity in the two$ groups was also very similar.

Sex of Infant.-The vaccinated women had $51(44.7 \%)$ male children and the controls had $236(52.6 \%)$; this suggests an alteration of sex ratio among the infants whose mothers were vaccinated. However, the numbers in the vaccinated group are small and the difference noted between the proportion of males in this group and the unvaccinated control group is of no statistical significance $\left(\chi^{2}=2.227 ; \mathrm{n}=1 ; 0.20>\mathrm{P}>0.10\right)$.

\section{Discussion}

Several studies have been carried out to determine whether or not smallpox vaccination of expectant mothers exerts a harmful influence on the foetus. Urner (1927) was of the opinion that vaccination in pregnancy had no injurious effects on mothers or their infants, but all except one of the 129 expectant mothers were vaccinated after the third month of pregnancy. Bellows et al. (1949), from a study of 893 pregnant women, 720 of whom were vaccinated and 173 unvaccinated, considered that smallpox vaccination during pregnancy did not increase the incidence of congenital abnormalities, stillbirths, abortions, or infant deaths. Although the study was a prospective one the control group was very small, and the abortions, stillbirths, and malformations were not analysed according to the stage of pregnancy in weeks when vaccination was performed. Greenberg et al. (1949) observed 4,172 women vaccinated in the first trimester of pregnancy. A control unvaccinated group included 2,186 women in the same period of pregnancy. No difference was demonstrated between the two groups for malformed infants, prematurity, or stillbirths. They concluded that smallpox vaccination of women in the first trimester of pregnancy did not exert any deleterious effect on the developing embryo. This was a retrospective study, and is more liable to error than a prospective study; further, no information was available on abortions.

MacArthur (1952) described a case of congenital vaccinia and then studied a series of pregnant women vaccinated at the time of an outbreak of smallpox in the West of Scotland to determine the frequency with which vaccination during pregnancy had a harmful effect on the foetus. He forwarded a questionary to 5,059 women under the age of 45 years who had given the title of "Mrs." before their name at the time of vaccination. The Post Office delivered the questionary to 4,827 of these women, $3,408(70.6 \%)$ replies being received. $\mathrm{He}$ argues with some conviction that the results of the study are unlikely to be biased by those who did not reply. The results show that vaccination of a pregnant woman during the first trimester of pregnancy, and especially during the second and third months of pregnancy, significantly increases foetal mortality. Again, this study was of retrospective type 
and no control unvaccinated series was available for comparison; the frequency of abortion among vaccinated mothers was compared with the highest incidence of foetal loss that Randall et al. (1950) considered could occur in a series of normal pregnancies.

Bieniarz and Dabrowski (1956) compared the outcome of pregnancy in 1,270 vaccinated and 3,515 unvaccinated pregnant women in Poland. They showed that the incidence of abortion in women vaccinated up to the end of the sixteenth week of pregnancy was significantly higher than that of the controls, and they reported no increase in congenital malformations. The numbers are certainly impressive, but some facts about the study detract from its value; it is not stated whether or not the vaccinated women had successful reactions, and, furthermore, the cases and controls might not have been exposed to the same environmental influences. While the cases related to women who were pregnant in 1953, the great majority of the controls $(2,822,80.3 \%)$ were women who were pregnant in the preceding year.

The prospective type of inquiry for cases and controls is the only satisfactory approach to the problem of the effects of virus infections during pregnancy (Logan, 1951). In the present prospective study the outcome of the pregnancies of 112 mothers successfully vaccinated against smallpox in the course of pregnancy is compared with a control group of 448 mothers who were unvaccinated and pregnant during the same time. The results do not indicate that vaccination exerted a harmful influence on the foetus for the factors examined, but admittedly the number of vaccinated women in the study is small. The incidence of abortion among the vaccinated and control groups is identical. MacArthur (1952) reported that almost $30 \%$ of pregnancies where vaccination was performed within 4 to 12 weeks of the beginning of pregnancy resulted in abortion. This was not demonstrated in the present study, in which 43 women were vaccinated when between 4 and 12 weeks pregnant and only one abortion occurred, 16 weeks later; on the figures quoted by MacArthur one would have expected approximately 13 abortions in this group. It should be mentioned that at least some weeks elapsed between the inception of the vaccination campaign and the beginning of the study. It is possible that women vaccinated early in pregnancy who aborted soon afterwards could have escaped inclusion in the study. However, as pointed out, only one abortion occurred among 43 women vaccinated when between 4 and 12 weeks pregnant, almost certainly suggesting that if vaccination at this stage of pregnancy is associated with abortion the incidence cannot be as high as that quoted by MacArthur (1952).

There is no doubt that primary vaccination in early or midpregnancy may, albeit rarely, produce a foetal vaccinia which will result in death of the foetus. Such cases have been reported by MacDonald and MacArthur (1953), Tucker and Sibson (1962), Entwistle et al. (1962), and Naidoo and Hirsch (1963) among others. Wielenga et al. (1961) also reported a case of prenatal vaccinia where the mother was unvaccinated, and they concluded that she had received an airborne vaccinia infection from her recently vaccinated son. With the modern need for vaccination against smallpox for intended travel to many countries, coupled with the fact that a woman may be unaware that she is pregnant at the time of vaccination, one could argue in favour of vaccinating all female infants at the age of minimum risk (1-4 years) and revaccinating within intervals of ten years when complications of a serious nature are apparently negligible (Bourke, 1964); congenital vaccinia has not yet been reported in a pregnant woman with a previous history of successful vaccination.

\section{Summary}

In a prospective study the results of the pregnancies of 112 women vaccinated during their pregnancies were compared with the outcome of the pregnancies of 448 controls. No statistically significant differences were noted for pregnancy duration, abortions, stillbirths, neonatal deaths, congenital malformations, or the weights and sex of the infants.

It is pointed out that, as the rare condition of prenatal vaccinia may follow primary vaccination in pregnancy, this procedure should never be carried out on pregnant women as a routine.

We are indebted to the Masters of the Coombe, National Maternity, and Rotunda Hospitals, and the consultant obstetrician of St. Kevin's Obstetric Hospital and their staffs in the out-patient and medical records departments for their co-operation and assistance. We are grateful to Mrs. E. McSweeney for clerical help.

\section{REFERENCES}

Bellows, M. T., Hyman, M. E., and Merritt, K. K. (1949). Publ. Hlth Rep. (Wash.), 64, 319.

Bieniarz, J., and Dabrowski, Z. (1956). Pol. Tyg. lek., 2, 2183.

Bourke, G. J. (1964). Publ. Hith (Lond.), 78, 84.

Entwistle, D. M., Bray, P. T., and Laurence, K. M. (1962). Brit. med. f., 2, 238.

Greenberg, M., Yankhauer, A., Krugman, S., Osborn, J. J., Ward, R. S., and Dancis, J. (1949). Pediatrics, 3, 456.

Gregg, N. McA. (1941). Trans. ophthal. Soc. Aust., 3, 35.

Logan, W. P. D. (1951). Brit. med. f., 2, 641.

MacArthur, P. (1952). Lancet, 2, 1104.

MacDonald, A. M., and MacAithur, P. (1953). Arch. Dis. Childh., 28, 311.

Naidoo, P., and Hirsch, H. (1963). Lancet, 1, 196.

Randall, C. L., Baetz, R. W., Hall, D. W., and Birtch, P. K. (1950). Cited by MacArthur (1952).

Tucker, S. M., and Sibson, D. E. (1962). Brit. med. f., 2, 237.

Urner, J. A. (1927). Amer. F. Obstet., 13, 70.

Wielenga, G., van Tongeren, H. A. E., Ferguson, A. H., and van Rijssel, Th. G. (1961). Lancet, 1, 258. 